\title{
Design and simulation of near-field waveguide probe fixed with circular SRR array
}

\author{
Siming Zhao a and Bing $\mathrm{Hu}$ \\ School of Information and Electronics, Beijing Institute of Technology, China
}

\begin{abstract}
The probe structure is the primary issue for near field detection procedure. In this paper, a waveguide probe with circular split ring resonator (SRR) array fixed on its open end is proposed. The probe explores the under-detected object surface by measuring the phase of $S_{11}$, which is quite sensitive to the standoff distance between probe and surface. Simulating results verify the probe to be an excellent near-field detecting instrument in lateral and depth resolution. Keywords: metallic surface crack detection; near-field probe; subwavelength resolution; split ring resonator.
\end{abstract}

\section{Introduction}

Due to the sensitivity of the change of evanescent electromagnetic field, microwave near-field probes are able to break through the classical Abbé limit and widely used for detecting or imaging the surface and sub-surface of objects. Many delicate designs were proposed, such as open-ended coaxial line probe in Ref. [1], open-ended dielectric slab-loaded waveguide probe in Ref. [1].With the introduction of the superlens concept proposed by Pendry [3], some periodically structured probes have become new alternative for near-field detection and imaging, including metal wire gratings described in Ref. [4], transmission-line lens in Ref. [5], concentrically corrugated near-field plate in Ref. [6], end-fire antenna array coaxial line probe in Ref. [7], dipole array probe in Ref. [8] and small loop coupled with rectangle SRR in Ref. [9, 10].

Most detecting technology of current microwave near-field probes utilizes the reflecting power from the under-detected surface. However, some researchers are engaged in exploring the surface or subsurface using phase shift of $S_{11}$ by inserting double (DNG) or single (SNG) negative media between the probe and under detecting surface[9,10]. In these researches, a rectangle small loop in Ref. [10] or circular small loop in Ref. [9] is used as the near field generator and single rectangle SRR in Ref. [9] or SRR array in Ref. [10] is used as $\mu$-negative media to enhance the near field and increase the phase shift. Numerical and experimental results proved these structures to be more sensitive than those without SRR, especially in the aspect of depth resolution.

In this paper, a near-field waveguide probe is proposed by fixing an array of circular SRR at the open end of a rectangle waveguide. In this structure the additional circular SRR array works by exerting the merits of $\mu$-negative media. The SRR array can easily be fabricated by printed circuit board (PCB) technique. The working frequency and the number of levels of the SRR array are discussed according to

a Corresponding author : classfourzsm@163.com

(C) 2016. The authors - Published by Atlantis Press 
numerical experiments by using Ansoft HFSS. Simulating results indicate the proposed probe has higher resolution in both lateral and depth directions than a waveguide probe without SRR array.

\section{Design of probe}

\subsection{Probe structure}

Fig. 1 shows the geometrical structure of the near-field rectangular waveguide probe fixed with an array of circular SRR in the open end of the waveguide.

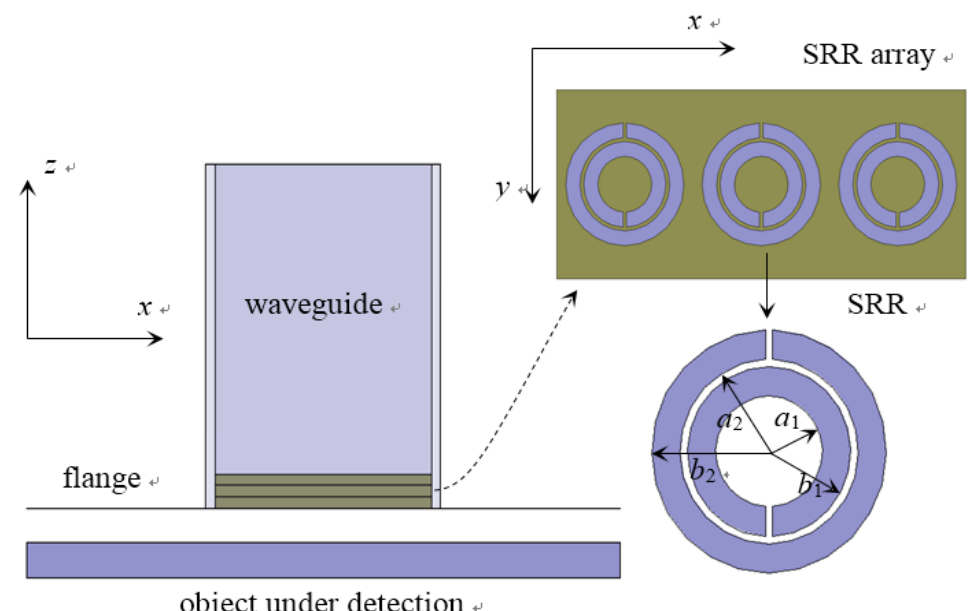

Figure 1. Geometrical structure of the near-field probe

The waveguide is a WR-90 type rectangular waveguide, which has a cross section of $22.86 \mathrm{~mm} \times 10.16 \mathrm{~mm}$ and a working band of $8.20 \sim 12.5 \mathrm{GHz}$ at $\mathrm{TE}_{10}$ mode. The classical circular SRR is selected as the cell of $\mu$-negative material. The SRR array is bolstered by a serial of low loss substrate slabs which have the same cross section of the waveguide and the typical thickness of $0.6 \mathrm{~mm}$ for single level PCB. The substrate material is Rogers 5880 with a relative permittivity of 2.2 and a dielectric loss tangent of 0.0009 . The inner and outer radii of the inner circular strip are $a_{1}=1.5 \mathrm{~mm}$ and $b_{1}=2.3 \mathrm{~mm}$ respectively, and $a_{2}=2.5 \mathrm{~mm}, b_{2}=3.3 \mathrm{~mm}$ for the outer strip. The gap width of both circular strip is $\mathrm{w}=0.2 \mathrm{~mm}$. At the same level, three SRRs are arranged and separated at same distance, in detail, the center of the middle one is located at the origin of $x-y$ plane, and the centers of the other two are at the distance of 7.62mm from the center of the middle one symmetrically. The SRR levels and substrate slabs construct a sandwich-like structure. Every level of substrate slab inserts between two levels of SRR, therefore, the number of SRR-level is one greater than the one of substrate-level. Besides, the probe has a metal flange which extends $20 \mathrm{~mm}$ from the four sides of the open end of the waveguide. Copper is assigned to be the constituent material of the waveguide, SRR and the flange structure.

\subsection{Working frequency selected}

As for the design on metamaterial, the electromagnetic characteristics of the $\mu$-negative material are determined when the substrate material and the geometric parameters of the SRR and substrate slab are appointed. However, some characters may change when the $\mu$-negative material is fixed in the waveguide open end because of the specific characters of the $\mathrm{TE}_{10}$ electromagnetic wave propagating in rectangle waveguide. For this issue, the proper working frequency should be chosen in the neighborhood of center frequency of waveguide. 


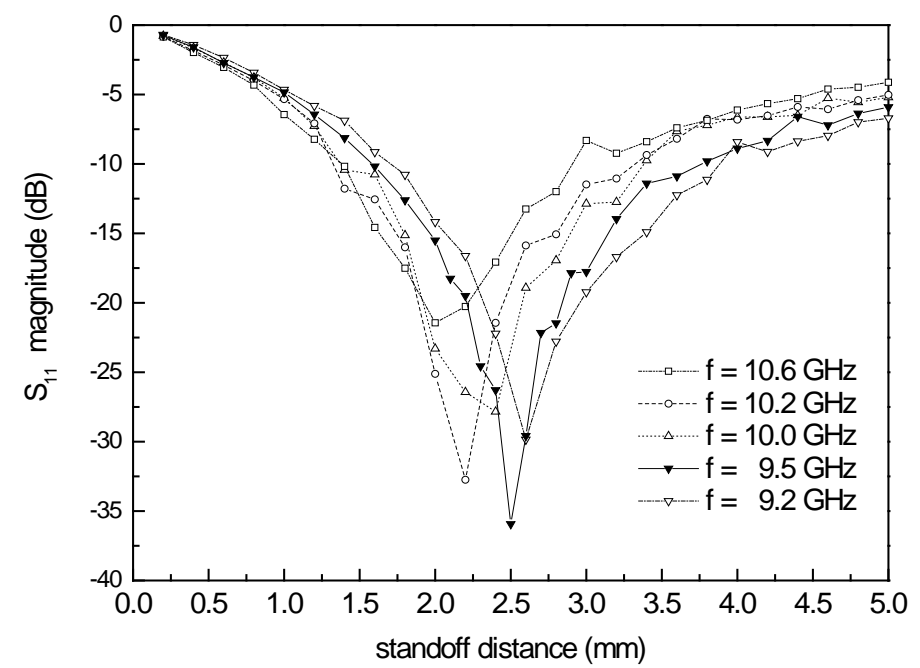

Figure 2. Magnitude of $S_{11}$ vs. standoff distance at different frequency. Magnitude of $S_{11}$ changes nonlinearly with standoff distance and reaches minimum at a certain distance. The curve at the frequency of $9.5 \mathrm{GHz}$ experiences a most drastic shift in the vicinity of $2.5 \mathrm{~mm}$.

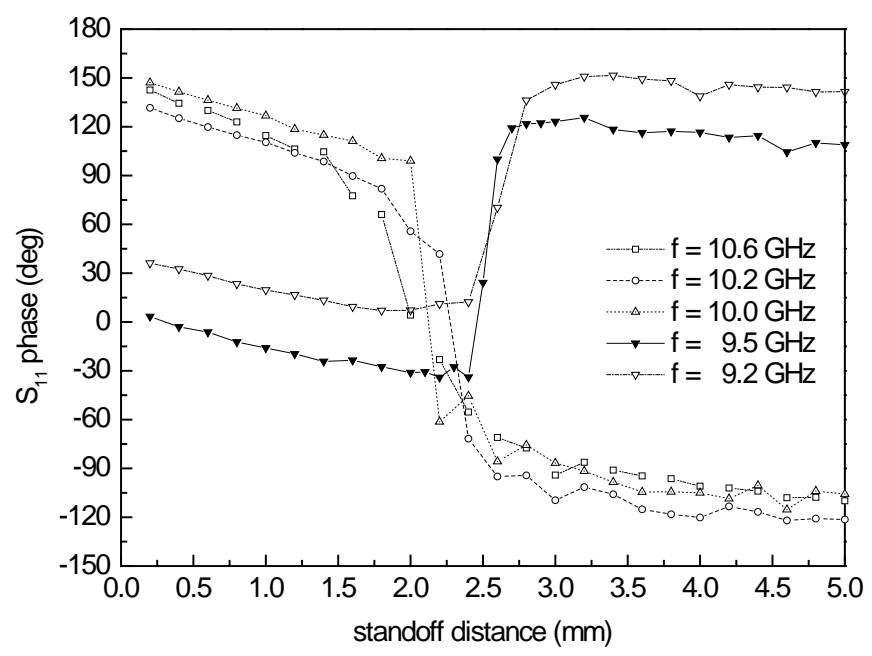

Figure 3. Phase of $S_{11}$ vs. standoff distance at different frequency. Phase of $S_{11}$ changes drastically with distance within a certain range of standoff distance. The curve at also the frequency of $9.5 \mathrm{GHz}$ experiences a most drastic shift in the vicinity of $2.5 \mathrm{~mm}$.

The simplest structure of two levels of SRR and one level of substrate (2-SRR\&1-Sub) is investigated and the under detected object is an infinite perfect surface in simulation procedure. Fig. 2 and 3 show the curves of $S_{11}$ magnitude and phase vs. the standoff distance between probe and object at different working frequency. From the two plots, it can be seen that the resonance effect is very clear at 9.5GHz. Namely, the probe has the minimum $S_{11}$ magnitude and the most distinct phase change when $d=2.5 \mathrm{~mm}$ at $9.5 \mathrm{GHz}$. Therefore, $9.5 \mathrm{GHz}$ is selected to be the working frequency at which the SRR array 
acts as a sensitive phase shift amplifier.

\subsection{Status of different levels}

Except for providing the clue to determine the working frequency, different curves in Fig. 2 and 3 also show serious fluctuant phenomena especially at the neighborhood of resonance point. Therefore, more structures are compared for selecting for fluent curve of phase vs. standoff distance.

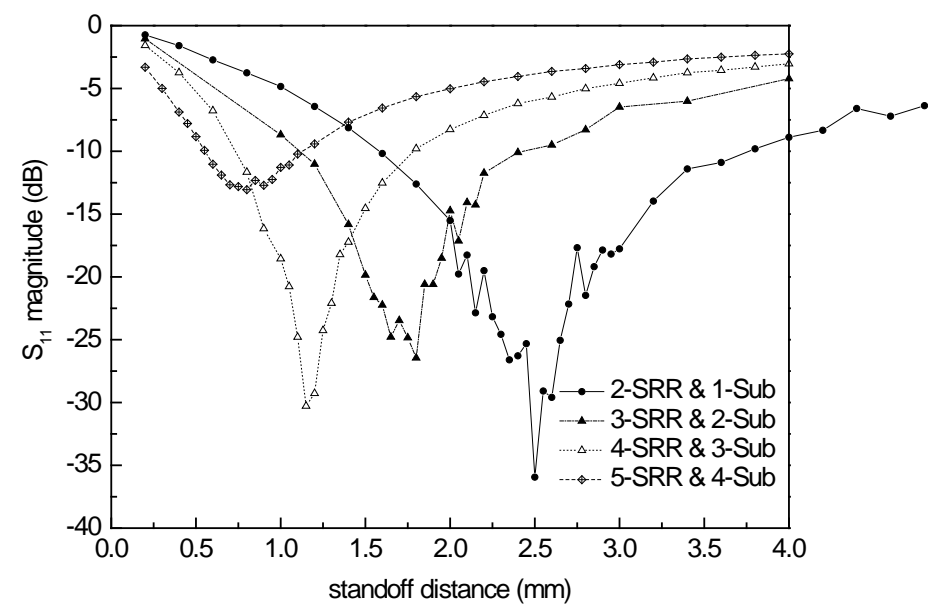

Figure 4. Magnitude of $\mathrm{S}_{11}$ vs. standoff distance for different structure.

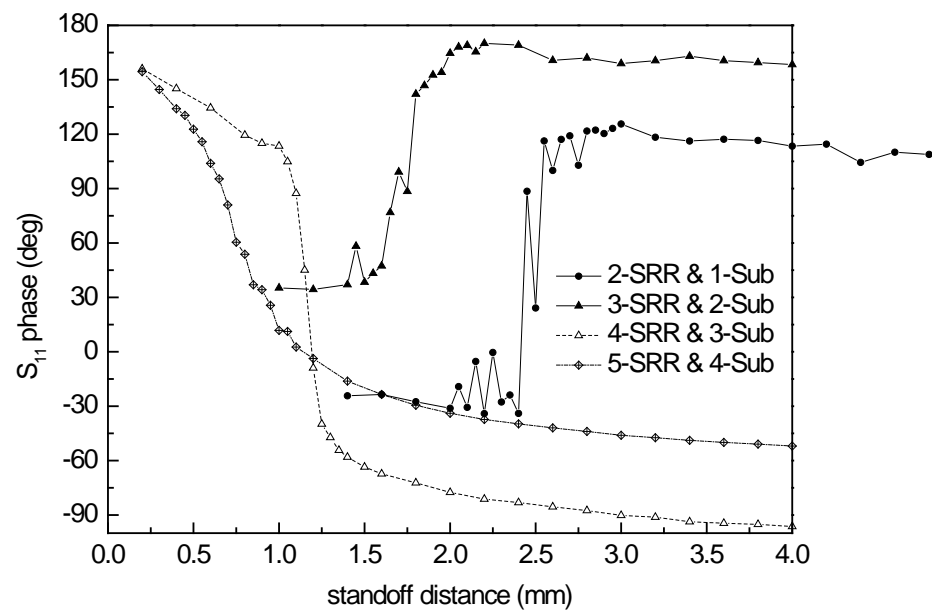

Figure 5. Phase of $\mathrm{S}_{11}$ vs. standoff distance for different structure.

Three structures are compared above to select a probe which has smooth response to the standoff distance between probe and surface of object at the operating frequency of 9.5GHz. The three structures are 2-SRR\&1-Sub, 3-SRR\&2-Sub and 4-SRR\&3-Sub.

According to the curves in Fig. 4 and 5, resonance positions change corresponding to different structures. Besides, the resonance standoff distance is smaller, at which the curves become smoother 
with more levels of SRR and substrate introduced. Especially, the curve of 4-SRR\&3-Sub hardly shows fluctuant. Though more levels of SRR and substrate can give more smooth response curve, it may introduce more reflection which can be verified by Fig. 4 in which the magnitude of $S_{11}$ for 5-SRR\&4-Sub is much greater than other cases. Therefore, the 4-SRR\&3-Sub structure is ultimately selected to be fixed at the waveguide open end.

\section{Depth and lateral resolution}

Numerical experiment instruments for investigating the depth resolution are shown in Fig. 6, in which the under detected target is assigned to be an aluminum slab which has the thickness of 3mm and two steps with height of 0.3 and $0.6 \mathrm{~mm}$ respectively. The edges of two steps are parallel to y-axis and depart $15.8 \mathrm{~mm}$ from each other. In numerical simulation, the center of the two steps is on origin and the probe move from $-24 \mathrm{~mm}$ to $24 \mathrm{~mm}$ along $\mathrm{x}$-axis.

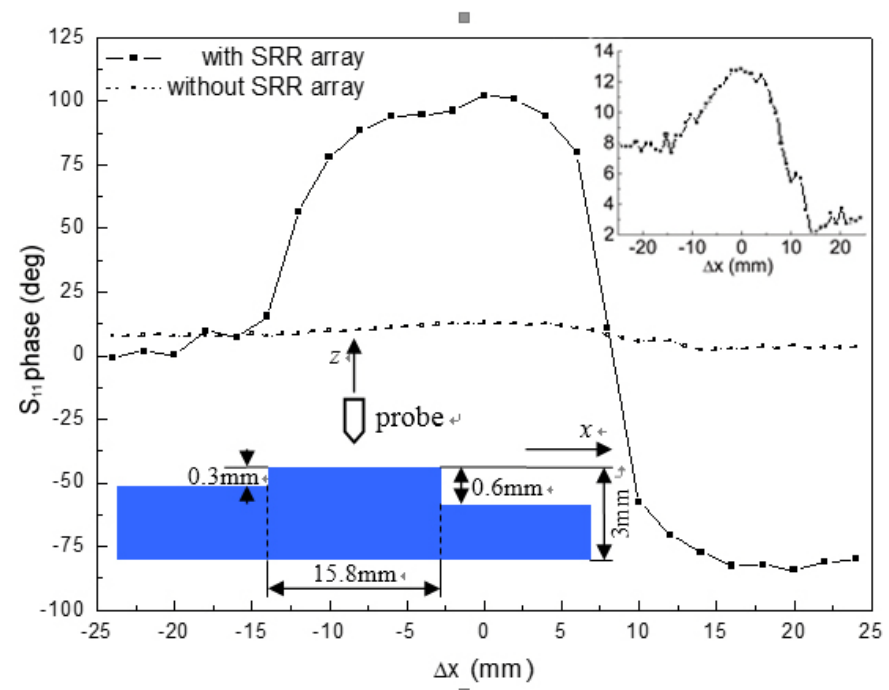

Figure 6. Comparison of depth resolution for the proposed probe and a waveguide without SRR array. Phase shift of probe with SRR is much higher than that of probe without SRR.

Fig. 6 clearly proves the proposed probe is superior to the one without SRR in depth resolution, for it exhibits the phase change of about $180^{\circ}$ for the detected structure, while the one without SRR shows only $12^{\circ}$.

To investigate the lateral resolution, the under detected target is assigned to be a simple PCB shown in Fig. 6, on which are two narrow copper strips whose width and length are $0.05 \lambda$ and $0.253 \lambda$ at $9.5 \mathrm{GHz}$ respectively. The substrate slabs have a thickness of $0.6 \mathrm{~mm}$ and the constituent material of Rogers 5880 . The couple of strips are located along $\mathrm{y}$-axis and under the probe at the distance of $1.2 \mathrm{~mm}$ where the probe is most sensitive to metal according to Fig. 5. In numerical simulation, the center of the strip couple is on origin and the probe move from $-0.5 \lambda$ to $0.5 \lambda$ along $x$-axis. In Fig. $6, s$ is defined as the separation between the two copper strip and $\Delta x$ is the distance of the probe center apart from the center of the strip couple.

Fig. 7 shows the lateral resolution for different probes. Normalized phase shift is defined as $\left(\varphi-\varphi_{\min }\right) /$ $\left(\varphi_{\max }-\varphi_{\min }\right)$, wherein $\varphi$ is the phase of $\mathrm{S}_{11}$. Fig. 7 indicates that two obvious peaks can be observed in the curve of probe with SRR when $s=0.33 \lambda$ and $s=0.4 \lambda$, while two distinct peaks in the curve of the probe without SRR do not exist at the same time. 

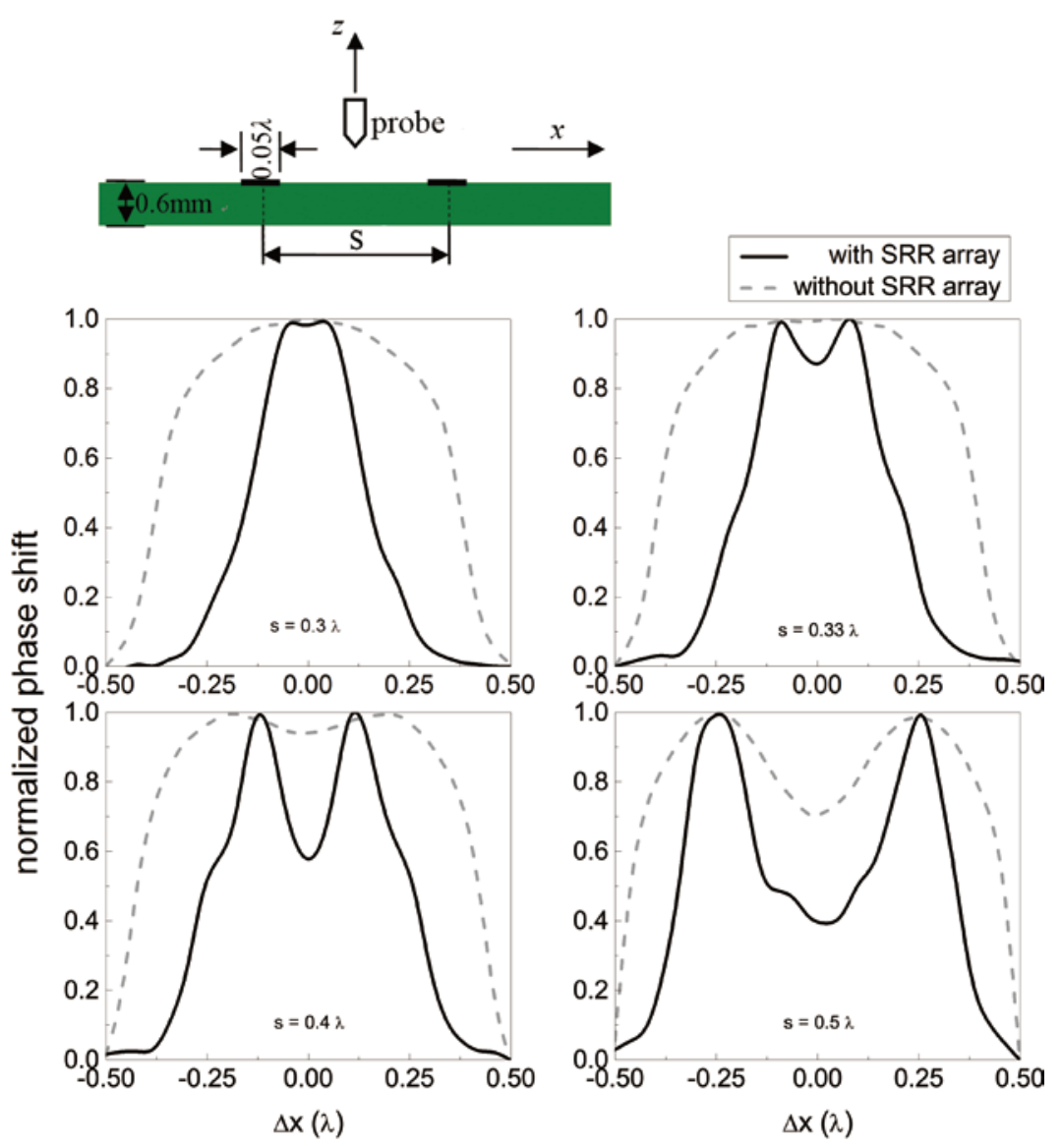

Figure 7. Comparison of lateral resolution for the proposed probe and a waveguide without SRR array.

\section{Conclusion}

The phase-sensitive near field probe is designed by fixing the circular SRR array on the rectangular waveguide open end. The steep and smooth response curve is achieved in the neighborhood of detection position by investigating the different structure at the selected working frequency. Simulating results indicate that the proposed probe exhibits obvious advantages in the surface detection procedure and potential application in PCB or rough metal surface investigation comparing to the only waveguide situation.

\section{References}

1. Y. Gao, A. Lauer, Q. Ren, I. Wolff, IEEE Trans. Microw. Theory Tech., 46, 1694-1703(1998)

2. M. T. Ghasr, S. Kharkovsky, R. Zoughi, R. Austin, IEEE Trans. Instrum. Meas., 54, 1497-1504, (2005)

3. J. B. Pendry, Phys. Rev. Lett., 85, 3966-3969(2000)

4. G. Fedorov, S. I. Maslovski and A. V. Dorofeenko, Phys. Rev. B, 73(2006)

5. J. Zhu, G. V. Eleftheriades, Phys. Rev. Lett., 101(2008)

6. M. F. Imani, A. Grbic, IEEE Trans. Microw. Theory Tech., 58, 3982-3988(2010)

7. L. Markley, G. V. Eleftheriades, IEEE Antennas Wireless Propag. Lett., 8, 1025-1028(2009) 
8. L. Markley, G. V. Eleftheriades, IEEE Trans. Microw. Theory Tech., 58, 551-558(2010)

9. M. S. Boybay, O. M. Ramahi, IEEE Trans. Microw. Theory Tech., 57, 3427-3433(2009)

10. Z. Ren, M. S. Boybay, O. M. Ramahi, IEEE Trans. Microw. Theory Tech., 59, 488-495(2011)

11. M. S. Boybay, O. M. Ramahi, Phys. Rev. E, Stat. Phys. Plasmas Fluids Relat. Interdiscip. Top., 79(2009) 